\title{
Effects of an Asthma Self-Management Support Service Provided by Community Pharmacists: A Systematic Review and Meta-Analysis
}

\author{
Suthasinee Dokbua, PharmD; Piyameth Dilokthornsakul, PharmD, PhD; \\ Nathorn Chaiyakunapruk, PharmD, PhD; Bandana Saini, PhD; Ines Krass, BPharm, PhD; \\ and Teerapon Dhippayom, PharmD, PhD
}

\begin{abstract}
BACKGROUND: Current evidence of the effects of pharmacy services on asthma outcomes are not conclusive, since most pharmacy services comprise a variety of interventions.

OBJECTIVE: To assess the effect of a service containing self-management support delivered by community pharmacists to patients with asthma.

METHODS: A systematic search was performed in the following databases from inception to January 2017: PubMed, Embase, Cochrane Library's Central Register of Controlled Trials, CINAHL (Cumulative Index to Nursing and Allied Health Literature) Plus, International Pharmaceutical Abstracts, and PsycInfo. Original studies were selected if they met the following criteria: (a) provided by community pharmacists; (b) the intervention service included the essential components of asthma self-management; (c) included a usual care group; and (d) measured control/severity of asthma symptoms, health-related quality of life (HRQOL), or medication adherence.
\end{abstract}

RESULTS: Of the 639 articles screened, 12 studies involving 2,121 asthma patients were included. Six studies were randomized trials, and the other 6 were nonrandomized trials. Patients with asthma who received a self-management support service by community pharmacists had better symptom control/lower severity compared with those receiving usual care (standardized mean difference [SMD] $=0.46 ; 95 \% \mathrm{Cl}=0.09-0.82$ ) with high heterogeneity $\left(I^{2}=82.6 \% ; P=0.000\right)$. The overall improvement in HRQOL and medication adherence among patients in the asthma self-management support group was greater than for those in the usual care group with SMD of $0.23(95 \% \mathrm{Cl}=0.12-0.34)$ and $0.44(95 \% \mathrm{Cl}=0.27-0.61)$, respectively. Evidence of heterogeneity was not observed in these 2 outcomes.

CONCLUSIONS: Self-management support service provided by community pharmacists can help improve symptom control, quality of life, and medication adherence in patients with asthma.

J Manag Care Spec Pharm. 2018;24(11):1184-96

Copyright @ 2018, Academy of Managed Care Pharmacy. All rights reserved.

\section{What is already known about this subject}

Community pharmacy services, which comprise a variety of interventions, are shown to have positive effects on asthma control. Self-management is one of the key elements for effective asthma control and is recommended in most asthma guidelines across the world.

\section{What this study adds}

This study focused on asthma self-management support services to make it more rational to combine results across studies of community pharmacy service with a similar concept and components. Asthma self-management support services provided by community pharmacists were shown to improve asthma control, quality of life, and medication adherence in adult patients with asthma. To achieve better asthma control, community pharmacists should provide self-management support that comprises self-monitoring and an action plan, incorporates additional services to identify and resolve drug therapy problems, and focuses on patients with poorly controlled asthma.

A sthma is a common but serious chronic respiratory disease worldwide. Through recent decades, asthma prevalence has been increasing, with an estimated over 200 million people reporting asthma globally. ${ }^{1}$ Asthma symptoms include cough, wheeze, and shortness of breath; chest tightness; and expiratory airflow limitation. A number of indicators have been used to measure success in asthma management such as asthma control/symptom severity, health-related quality of life (HRQOL), and adherence to asthma medication. Asthma is highly variable-although asthma symptoms may have been absent for a period of time, patients can experience exacerbations that may be life-threatening, especially among those with poor asthma control.

Effective asthma control requires appropriate medication, effective monitoring, patient education, and adherence to therapy. Failure to avoid trigger factors and inadequate adherence either due to not using medication as prescribed or incorrect inhalation technique would lessen the efficacy of controller medication and, in turn, minimize asthma control. A key strategy that may improve asthma control is to provide patients with the knowledge and skills to effectively manage their asthma. According to the Global Initiative for Asthma report, guided asthma self-management education and skills training should include several elements: skills training to use inhaler devices effectively, facilitation of adherence to treatment and management advice, asthma information provision, training on self-monitoring of symptoms or lung function, a written 
asthma action plan to guide self-management, and regular reviews by a health care provider. Self-management education is highlighted in most asthma guidelines across the world. ${ }^{2-4}$

To date, a body of evidence supports the benefits of a number of self-management support interventions on asthma symptoms, unscheduled health care use, medication adherence, and general health-related and asthma-specific quality of life. ${ }^{5,6}$ In particular, a recent systematic meta-review consolidated this evidence. ${ }^{7}$ Although this meta-review suggested that support for self-management of asthma is applicable in a broad range of clinical settings, it is unclear if this service intervention can be delivered with similar beneficial effects in a specific setting such as community pharmacy.

Community pharmacy is an ideal setting for health care service delivery because it is considered one of the most accessible health care sites. To date, there is reasonable evidence of positive effects of pharmacy services on asthma control in many large-scale randomized trials. ${ }^{89}$ However, systematic reviews conducted to estimate pooled effects of pharmacy services on asthma outcomes cannot attribute improvements to any one service element, as most pharmacy services comprise a mix of varied interventions such as self-management education, lung function testing, medication review, and doctor referrals. ${ }^{10,11}$

The current review has focused on one of the most important elements of pharmacy care-asthma self-management support services-to make it more rational to statistically pool results across studies of service with a similar concept and components. Adverse effects of this asthma self-management support service were also determined as an outcome of interest. The aim of this systematic review was to assess the effects of a service containing self-management support provided by community pharmacists to patients with asthma compared with usual care.

\section{Methods}

This systematic review was conducted according to the Cochrane Effective Practice and Organisation of Care (EPOC) guidance, ${ }^{12}$ and the reporting follows the PRISMA Statement. ${ }^{13}$

\section{Inclusion Criteria}

Research articles included in this review were those that reported the effects of self-management support interventions provided to patients with asthma compared with a separate control group. Full-text studies of randomized trials, nonrandomized trials, and controlled before-after studies were included if they met the following inclusion criteria: (a) provided by a community pharmacist in community pharmacy settings; (b) included essential components of guided asthma self-management (i.e., patient self-monitoring, provision of written asthma action plan, or goal setting); (c) compared a self-management support service provided by a community pharmacist with standard or usual care group; and (d) measured one of the following patient outcomes: asthma symptom control/severity, HRQOL, or medication adherence. Studies were included irrespective of their publication status and language of publication.

\section{Data Sources and Search Strategies}

The following databases were used to search for original research articles: PubMed, Embase, CENTRAL (Cochrane Library hosts the Central Register of Controlled Trials), CINAHL (Cumulative Index to Nursing and Allied Health Literature) Plus, International Pharmaceutical Abstracts, and PsycInfo. Grey literature was searched from the National Technical Information Service, Open Grey, and Digital Access to Research Theses (DART)-Europe. Reference lists of all included studies, as well as relevant systematic reviews, were reviewed.

Strategic searches were conducted using keywords and controlled vocabulary terms, which included asthma and (a) community pharma*, or (b) independent pharma*, or (c) retail pharma*, or (d) chain pharma*. All databases were searched from database inception date to the date of search; searches were conducted between January 26 and 30, 2017.

\section{Search and Study Selection}

All titles and abstracts retrieved by electronic searching were downloaded to EndNote X7 (Clarivate Analytics, Philadelphia, PA). After duplicate removal, a reviewer (Dokbua) scanned all the titles and abstracts to determine whether the extracted studies assessed community pharmacy support services on asthma self-management. This initial screening process was checked by another reviewer (Dhippayom). The eligibility of full-text articles of selected studies was independently assessed by 3 reviewers (Dokbua, Dhippayom, and Saini). Disagreements and uncertainties regarding eligibility were resolved by discussion. Potential studies that failed to meet the inclusion criteria were grouped in accordance to the reported reasons for exclusion.

\section{Data Collection and Quality Assessment}

Data extraction was undertaken by 2 reviewers (Dokbua and Dhippayom) using a data collection form modified from the Cochrane EPOC guidelines. ${ }^{14}$ The data extracted and reported the following: study design; number of study centers and location; date of study; length of follow-up; randomization process; number of patients; age of patients; gender; inclusion and exclusion criteria; level of asthma control/symptoms; characteristics of the intervention (in terms of description of the intervention, number and duration of sessions provided, intervention span/ period and whether any form of training preceded the implementation of the intervention); outcomes of interest including beneficial effects (i.e., asthma control/symptoms, quality of life, medication adherence) and possible adverse outcomes (such as 
Effects of an Asthma Self-Management Support Service Provided by

\section{FIGURE 1 PRISMA Flow Diagram of Selected Articles}

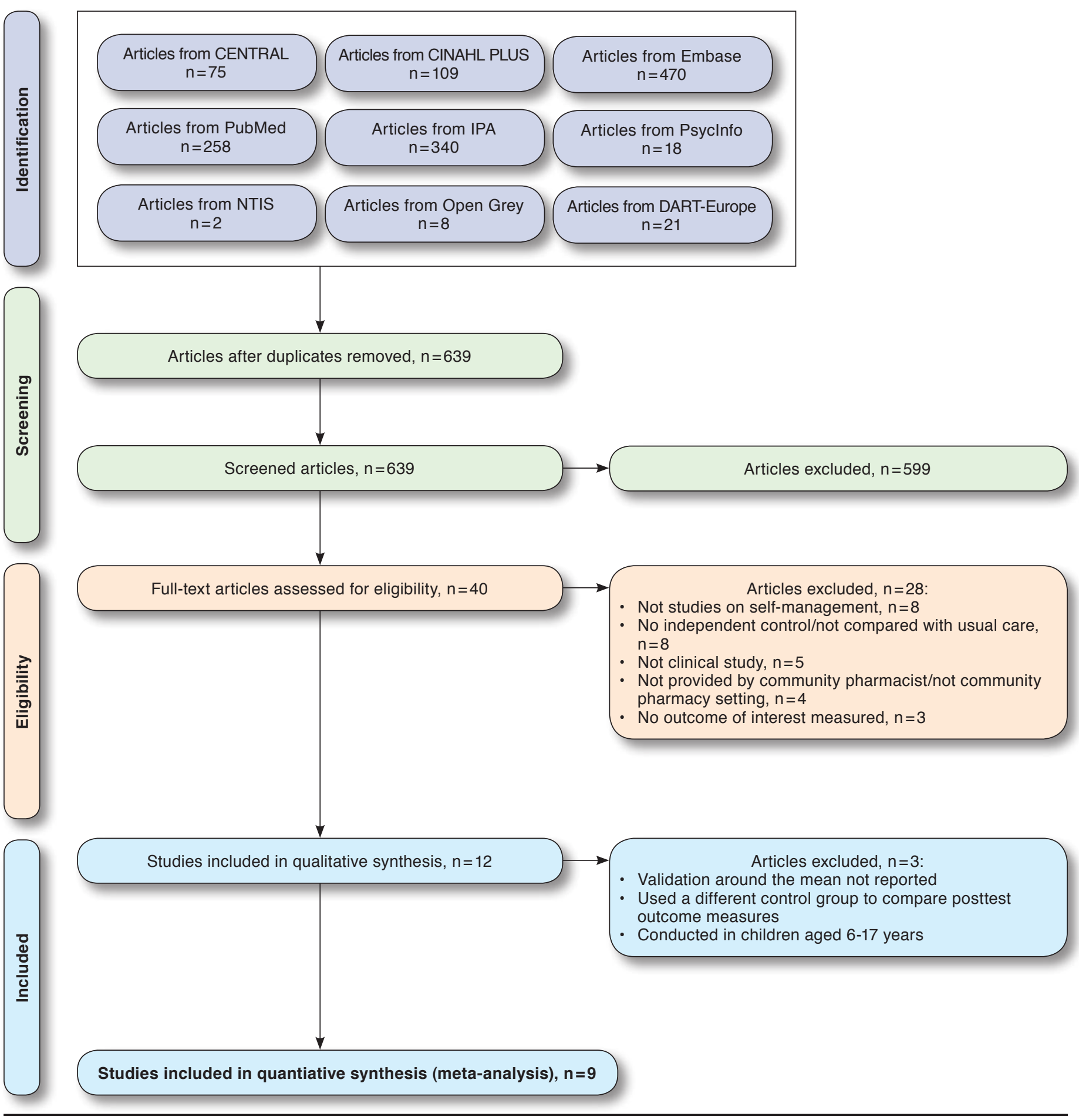

CINAHL = Cumulative Index to Nursing and Allied Health Literature; CENTRAL =Cochrane Library's Central Register of Controlled Trials; DART = Digital Access to Research Theses; IPA = International Pharmaceutical Abstracts; NTIS = National Technical Information Service. 
health behavior and health care utilization); funding; conflicts of interest of the authors; and ethical approval.

Studies included in this review were assessed for methodological quality by 2 reviewers (Dokbua and Dilokthornsakul) using the EPOC risk of bias tool, which was designed to measure the quality of studies on educational, behavioral, financial, regulatory, and organizational interventions. ${ }^{15}$ Studies in which training of participating pharmacists was not provided or reported were considered as high risk for the domain of "other risks of bias."

The summary assessment of the risk of bias within a study for a specific outcome was based on the risk of bias of key domains that, in this review, were "similar baseline characteristics," "protect against contamination," and "free from other risks of bias." The risk of bias summary for each study was classified as low risk (low risk of bias for all key domains), high risk (high risk of bias for 1 or more key domains), or unclear risk (unclear risk of bias for 1 or more key domains). ${ }^{16}$ Disagreements between reviewers were resolved by discussions with 1 of the other reviewers (Dhippayom).

\section{Outcome Measures}

The main outcome of interest in this review was clinical: the patient's asthma control/level of severity. Secondary outcomes of this study were (a) HRQOL; (b) health behavior with respect to medication adherence; and (c) adverse effects of the service (e.g., changes in health care utilization). Adverse effects of the selfmanagement support service, as suggested by EPOC, ${ }^{17}$ included adverse effects on health or health behavior; utilization, coverage or access; quality of care; resource use; health care providers (e.g., stress, burnout, or sick leave); social outcomes (e.g., poverty measures, employment, education); and equity.

\section{Synthesis of Results}

The findings were stratified based on specific outcome measures. Data from studies that reported outcomes of the same category were pooled in a meta-analysis to determine the overall effect size with a 95\% confidence interval (CI). Mean changes in the outcome variables for each study arm were calculated by subtracting baseline mean from postintervention mean. Pooled standard deviations of the mean changes were used. Then mean changes of the outcome variables were compared between intervention and comparator arms by calculating the overall mean differences. Since the true effects were expected to be related but not the same for included studies, a random-effects model was employed for all analysis (DerSimonian and Laird, $1986^{18}$ ).

Statistical heterogeneity between studies was assessed using the chi-squared test and $\mathrm{I}^{2}$. Thresholds of $\mathrm{I}^{2}$ were interpreted in accordance with the magnitude and direction of effects and strength of evidence of heterogeneity. ${ }^{19}$ Once statistical heterogeneity was found, potential sources of heterogeneity were explored by performing the following subgroup analyses: patients' asthma condition (uncontrolled vs. any level of asthma); component of the service (containing self-monitoring support and action plan/goal setting vs. containing only 1 of these components); study duration (short term, $\leq 6$ months vs. long term, $>6$ months); and type of additional service. Metaanalyses were conducted using Stata version 10.1 (StataCorp, College Station, TX).

\section{Results}

\section{Study Selection}

The search of published and grey literature in various databases yielded 639 articles after duplicates were removed (Figure 1). Of these, 40 articles were selected for full-text review based on titles and abstracts screened. A total of 28 papers were excluded after full-text review. Twelve studies met the inclusion criteria for this review. ${ }^{20-31}$ Of those studies, 3 were not included in the quantitative analysis because they did not report variation around the mean ${ }^{25}$; they used a different control group to compare posttest outcome measures ${ }^{27}$; and the intervention was conducted only in children aged 6-17 years. ${ }^{31}$ The latter study was not included for meta-analysis, since it focused on a different population with the remaining studies, which created clinical heterogeneity if combined with others. ${ }^{31}$

\section{Study Characteristics}

Of the 12 included studies, half were randomized trials, while the remaining were nonrandomized trials (Table 1). All studies were conducted in Western countries, with almost all of them conducted in developed (high-income) countries. These countries included Australia, Canada, Denmark, Germany, New Zealand, the United Kingdom, and the United States. There was, however, 1 study from an upper middle-income country (i.e., Bulgaria). Five studies measured only 1 main outcome: either the effect of a self-management support service on asthma control/severity (2 studies) or the effect on HRQOL (3 studies). Three studies explored the effect on both asthma symptom control/severity and HRQOL, and the remaining 4 studies reported medication adherence rates in addition to both outcomes. There was no study that investigated/reported adverse effects of the service.

Almost all of the included trials were conducted with an adult asthma population (mean age between 35.1 and 51.4 years). Only 1 study investigated the effect of pharmacy services in children with asthma. The number of patients participating in the included studies ranged from 24 to 500 . Eight studies recruited patients with uncontrolled or moderate to severe asthma.

The number of participating pharmacists in each study ranged from 1 to 57 . Community pharmacists in all 12 studies received training before the delivery of the corresponding interventions, although not much detail of the training elements was provided in some studies (Table 2). The duration of 


\begin{tabular}{|c|c|c|c|c|c|c|c|c|c|c|c|}
\hline$\stackrel{\hat{E}}{\tilde{E}}$ & $\widehat{\widehat{\Xi}}$ & 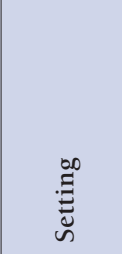 & 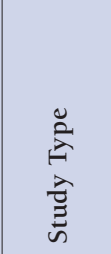 & 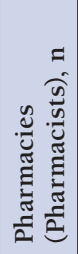 & 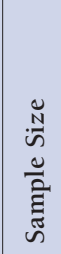 & 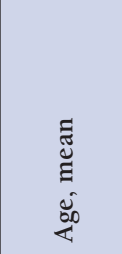 & 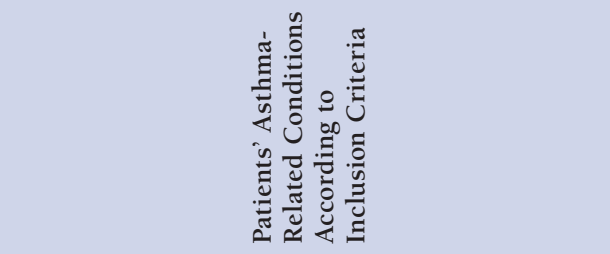 & 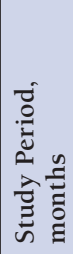 & $\begin{array}{l}\tilde{U} \\
\tilde{\Xi} \\
\stackrel{0}{\Xi} \\
0\end{array}$ & $\begin{array}{l}\overline{8} \\
0 \\
0 \\
\infty \\
\vdots \\
\vdots \\
0 \\
\tilde{J} \\
\Sigma\end{array}$ & 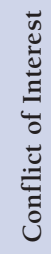 \\
\hline \multirow[t]{3}{*}{$\begin{array}{l}\text { Armour } \\
(2007)^{20}\end{array}$} & \multirow[t]{3}{*}{ Australia } & \multirow[t]{3}{*}{$\begin{array}{l}\text { Urban } \\
\text { and rural }\end{array}$} & \multirow[t]{3}{*}{ RT } & \multirow[t]{3}{*}{$\begin{array}{c}50 \\
(57)\end{array}$} & \multirow[t]{3}{*}{396} & \multirow[t]{3}{*}{$\begin{array}{l}\text { 47.5 Int, } \\
\text { 50.4 Ctrl }\end{array}$} & \multirow{3}{*}{$\begin{array}{l}\text { Had one of these conditions over the past } 4 \text { weeks: } \\
\text { used reliever medication }>3 \text { times a week, waking } \\
\text { at night or morning with cough/chest tightness } \\
\text { at least } 1 \text { occasion, time off work/study because } \\
\text { of asthma or symptoms of asthma at least once a } \\
\text { week; or no visit to a doctor for asthma within the } \\
\text { last } 6 \text { months }\end{array}$} & 6 & $\begin{array}{l}\text { Control/ } \\
\text { severity }\end{array}$ & \begin{tabular}{|c|} 
NAC \\
asthma \\
severity \\
assessment
\end{tabular} & \multirow[t]{3}{*}{ No } \\
\hline & & & & & & & & & QOL & AQLQ & \\
\hline & & & & & & & & & $\begin{array}{l}\text { Medication } \\
\text { adherence }\end{array}$ & BMQ & \\
\hline $\begin{array}{l}\text { Barbanel } \\
(2003)^{21}\end{array}$ & \begin{tabular}{|c|} 
United \\
Kingdom
\end{tabular} & Urban & RT & $\begin{array}{c}1 \\
(1)\end{array}$ & 24 & $\begin{array}{l}45 \mathrm{Int}, \\
47 \mathrm{Ctrl}\end{array}$ & $\begin{array}{l}\text { Used ICS; not recently attended secondary care } \\
\text { with asthma; had not changed asthma treatment in } \\
\text { the last } 6 \text { weeks; not recently had acute respiratory } \\
\text { infection }\end{array}$ & 3 & $\begin{array}{l}\text { Control// } \\
\text { severity }\end{array}$ & \begin{tabular}{|c|} 
North of \\
England \\
asthma \\
symptoms \\
scale
\end{tabular} & NR \\
\hline $\begin{array}{l}\text { Charrois } \\
(2006)^{22}\end{array}$ & Canada & Rural & RT & $\begin{array}{c}5 \\
\text { (NR) }\end{array}$ & 70 & $\begin{array}{l}\text { 38.7 Int, } \\
\text { 35.7 Ctrl }\end{array}$ & $\begin{array}{l}\text { High-risk asthma patients who had an ED visit or } \\
\text { hospital admission due to asthma in the previous } \\
12 \text { months or who had used more than } 2 \text { canisters } \\
\text { of inhaled } \beta 2 \text {-agonist in the previous } 6 \text { months }\end{array}$ & 6 & $\begin{array}{l}\text { Control/ } \\
\text { severity }\end{array}$ & ACQ & NR \\
\hline \multirow[t]{2}{*}{$\begin{array}{l}\text { Herborg } \\
(2001)^{23}\end{array}$} & \multirow[t]{2}{*}{ Denmark } & \multirow[t]{2}{*}{$\begin{array}{l}\text { Nation- } \\
\text { wide }\end{array}$} & \multirow[t]{2}{*}{ Non-RT } & \multirow[t]{2}{*}{$\begin{array}{c}31 \\
(31)\end{array}$} & \multirow[t]{2}{*}{500} & \multirow[t]{2}{*}{$\begin{array}{l}\text { 38.8 Int, } \\
\text { 42.4 Ctrl }\end{array}$} & \multirow[t]{2}{*}{ Moderate to severe asthma; using antiasthma drugs } & 12 & $\begin{array}{l}\text { Control/ } \\
\text { severity }\end{array}$ & $\begin{array}{c}\text { Asthma } \\
\text { morbidity } \\
\text { index }\end{array}$ & \multirow[t]{2}{*}{ No } \\
\hline & & & & & & & & & QOL & $\begin{array}{c}\text { NHP and } \\
\text { LWAQ }\end{array}$ & \\
\hline $\begin{array}{l}\text { Kheir } \\
(2001)^{24}\end{array}$ & $\begin{array}{c}\text { New } \\
\text { Zealand }\end{array}$ & $\mathrm{NR}^{\mathrm{a}}$ & Non-RT & $\begin{array}{c}5 \\
\text { (NR) }\end{array}$ & 62 & $\begin{array}{l}\text { 46.1 Int, } \\
\text { 44.5 Ctrl }\end{array}$ & $\begin{array}{l}\text { Had been diagnosed with asthma at least } 6 \\
\text { months; asthma condition was symptomatic and } \\
\text { not optimally controlled; no other comorbidity }\end{array}$ & 4 & QOL & $\begin{array}{c}\text { SF-36 and } \\
\text { AQLQ }\end{array}$ & NR \\
\hline \multirow[t]{2}{*}{$\begin{array}{l}\text { McLean } \\
(2003)^{25}\end{array}$} & \multirow[t]{2}{*}{ Canada } & \multirow[t]{2}{*}{$\mathrm{NR}^{\mathrm{b}}$} & \multirow[t]{2}{*}{ RT } & \multirow[t]{2}{*}{$\begin{array}{c}18 \\
(20)\end{array}$} & \multirow[t]{2}{*}{224} & \multirow[t]{2}{*}{48} & \multirow[t]{2}{*}{ Uncontrolled asthma } & \multirow[t]{2}{*}{12} & $\begin{array}{l}\text { Control/ } \\
\text { severity }\end{array}$ & $\begin{array}{l}\text { The symp- } \\
\text { tom scores } \\
\end{array}$ & \multirow[t]{2}{*}{$\mathrm{Nl}$} \\
\hline & & & & & & & & & QOL & \begin{tabular}{|c|} 
Juniper \\
question- \\
naire
\end{tabular} & \\
\hline $\begin{array}{l}\text { Petkova } \\
(2008)^{26}\end{array}$ & Bulgaria & Urban & RT & $\begin{array}{c}10 \\
\text { (NR) }\end{array}$ & 50 & $\begin{array}{l}\text { 35.1 Int, } \\
\text { 40.8 Ctrl }\end{array}$ & $\begin{array}{l}\text { Had been diagnosed with bronchial asthma; no } \\
\text { significant pulmonary disease }\end{array}$ & 4 & QOL & $\begin{array}{c}\text { Asthma } \\
\text { assessment } \\
\text { form }\end{array}$ & NR \\
\hline \multirow[t]{3}{*}{$\begin{array}{l}\text { Saini } \\
(2004)^{27}\end{array}$} & \multirow[t]{3}{*}{ Australia } & \multirow[t]{3}{*}{$\begin{array}{l}\text { Urban } \\
\text { and } \\
\text { suburban }\end{array}$} & \multirow[t]{3}{*}{ Non-RT } & \multirow[t]{3}{*}{$\begin{array}{c}\text { NR } \\
(25)\end{array}$} & 102 & $\begin{array}{l}43 \text { Int, } \\
52 \text { Ctrll, } \\
\text { and }\end{array}$ & $\begin{array}{l}\text { Excessive use of bronchodilator medications (over } \\
3 \text { times a week); frequent acute attacks; had no } \\
\text { major disease or terminal illness }\end{array}$ & 6 & $\begin{array}{l}\text { Control// } \\
\text { severity }\end{array}$ & $\begin{array}{c}\begin{array}{c}\text { Asthma } \\
\text { severity } \\
\text { score }\end{array} \\
\end{array}$ & NR \\
\hline & & & & & & 42 Ctrl2 & & & QOL & $\begin{array}{l}\text { Asthma- } \\
\text { related } \\
\text { QOL score }\end{array}$ & \\
\hline & & & & & & & & & $\begin{array}{l}\text { Medication } \\
\text { adherence }\end{array}$ & BMQ & \\
\hline $\begin{array}{l}\text { Saini } \\
(2008)^{28}\end{array}$ & Australia & Rural & Non-RT & $\begin{array}{l}\text { NR } \\
(20)\end{array}$ & 90 & $\begin{array}{l}\text { 50.8 Int, } \\
\text { 50.4 Ctrl }\end{array}$ & $\begin{array}{l}\text { Excessive use of bronchodilator medications (over } \\
3 \text { times a week); frequent acute attacks; had no } \\
\text { major disease or terminal illness }\end{array}$ & 6 & $\begin{array}{l}\text { Control// } \\
\text { severity }\end{array}$ & $\begin{array}{c}\text { Asthma } \\
\text { severity } \\
\text { score }\end{array}$ & No \\
\hline & & & & & & & & & QOL & \begin{tabular}{|c|} 
Asthma- \\
related \\
QOL score \\
\end{tabular} & \\
\hline & & & & & & & & & $\begin{array}{l}\text { Medication } \\
\text { adherence }\end{array}$ & BMQ & \\
\hline $\begin{array}{l}\text { Schulz } \\
(2001)^{29}\end{array}$ & Germany & Urban & Non-RT & $\begin{array}{c}48 \\
(\mathrm{NR})\end{array}$ & 164 & $\begin{array}{l}46.3 \mathrm{Int} \\
45.9 \mathrm{Ctrl}\end{array}$ & $\begin{array}{l}\text { Mild to severe asthma (scored 1-3 according to the } \\
\text { Medical Research Council Dyspnea Scale) }\end{array}$ & 12 & $\begin{array}{l}\text { Control/ } \\
\text { severity }\end{array}$ & $\begin{array}{c}\text { Asthma } \\
\text { severity } \\
\text { score }\end{array}$ & NR \\
\hline & & & & & & & & & QOL & $\begin{array}{c}\text { SF-36 and } \\
\text { LWAQ }\end{array}$ & \\
\hline
\end{tabular}




\section{TABLE 1 Characteristics of Included Studies (continued)}

\begin{tabular}{|c|c|c|c|c|c|c|c|c|c|c|c|}
\hline 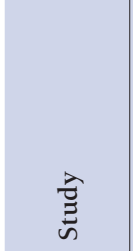 & 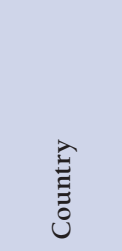 & 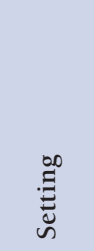 & 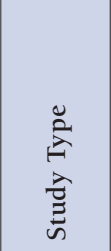 & 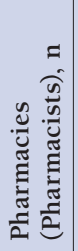 & 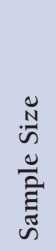 & 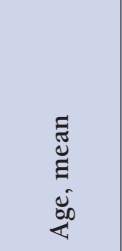 & 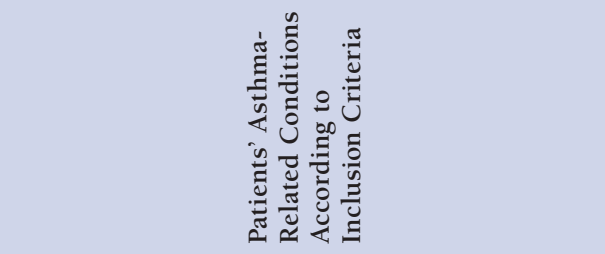 & 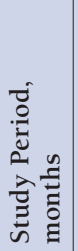 & 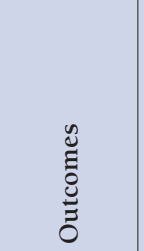 & 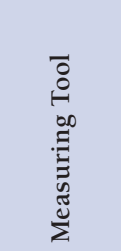 & 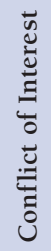 \\
\hline \multirow[t]{3}{*}{$\begin{array}{l}\text { Smith } \\
(2007)^{30}\end{array}$} & \multirow[t]{3}{*}{ Australia } & \multirow[t]{3}{*}{ Urban } & \multirow[t]{3}{*}{ Non-RT } & \multirow[t]{3}{*}{\begin{tabular}{c|}
21 \\
$(24)$
\end{tabular}} & \multirow[t]{3}{*}{109} & \multirow[t]{3}{*}{$\begin{array}{l}\text { 51.4 Int, } \\
\text { 54.5 Ctrl }\end{array}$} & \multirow[t]{3}{*}{ Previous use of preventer medication } & \multirow[t]{3}{*}{9} & $\begin{array}{l}\text { Control/ } \\
\text { severity }\end{array}$ & ACQ & \multirow[t]{3}{*}{ NR } \\
\hline & & & & & & & & & QOL & AQLQ & \\
\hline & & & & & & & & & $\begin{array}{c}\text { Medication } \\
\text { adherence }\end{array}$ & MARS & \\
\hline $\begin{array}{l}\text { Stergachis } \\
(2002)^{31}\end{array}$ & $\begin{array}{l}\text { United } \\
\text { States }\end{array}$ & Urban & RT & $\begin{array}{c}32 \\
(35)\end{array}$ & 330 & $\begin{array}{l}11.5 \mathrm{Int} \\
11.8 \mathrm{Ctrl}\end{array}$ & $\begin{array}{l}\text { Received either oral theophylline daily, oral or } \\
\text { inhaled } \beta 2 \text {-agonists more often than twice daily, or } \\
\text { inhaled anti-inflammatory agents for asthma daily }\end{array}$ & 12 & QOL & $\begin{array}{l}\text { Juniper } \\
\text { question- } \\
\text { naire }\end{array}$ & No \\
\hline
\end{tabular}

a Otago/Southland regions

${ }^{b}$ Health Outcome Pharmacies cooperative in British Columbia.

$A C Q=$ asthma control questionnaire; $A Q L Q=$ asthma quality of life questionnaire; $B M Q=b r i e f$ medication questionnaire; CoI = conflict of interest; $C$ trl $=$ control; $E D=$ emergency department; ICS = inhaled corticosteroids; Int=intervention; LWAQ = living with asthma questionnaire; MARS = Medication Adherence Report Scale; NAC=National Asthma Council; $N H P=$ Nottingham Health Profile; $N R=$ not reported; $Q O L=$ quality of life; $R T=$ randomized trial; SF-36=Short Form-36.

intervention sessions was reported in 7 studies with the average being 26.8 minutes to 1 hour. The duration of the entire intervention service in 7 studies was 6 months or less. During this period, pharmacists provided at least 4 sessions of intervention including follow-up visits. For the remaining 5 studies with services lasting longer than 6 months, the frequency of pharmacy services ranged from 6 to 12 sessions/follow-ups for up to 12 months after baseline.

Pharmacy services in all but 1 study were based on a guided intervention protocol, hence, the components of self-management support service given to patients in the same study were similar. The study by Smith et al. (2007) was the only study that provided individualized self-management support (i.e., delivered different service components to individual asthma patients depending on the areas of asthma control that were of personal relevance for each patient). ${ }^{30}$ Although the components of service varied across studies, the intervention in all of the included studies covered either self-monitoring or action plan provision/goal setting (Table 2). Few studies reported details of the action plans provided by or facilitated by community pharmacists. Self-monitoring was advocated in 9 studies where patients recorded their peak expiratory flow reading or symptoms in a diary on a routine basis or reported regularly to the pharmacists. Other additional services reported among the 12 studies included the following: identify, analyze, and resolve drug therapy problems (DTPs); a smoking cessation service; and optimization of drug therapy. Low fidelity to the service protocol by pharmacists was observed in 2 studies. Although the remaining 10 studies did not mention if participating pharmacists complied with the study protocol, some reported regular contact with the pharmacists to ensure that the service was conducted as planned.

\section{Quality of Included Studies}

The methodological quality assessment of the included studies is summarized in the Appendix (available in online article). Overall, pharmacies were the unit of allocation in 8 studies, ${ }^{20,23,25,27-31}$ while the remaining allocated intervention at the level of participating patients. ${ }^{21,22,24,26}$ For the 6 randomized trials, 3 studies randomized subjects to receive self-management support, which resulted in high risk of bias for contamination of receiving interventions in the control group. ${ }^{21,22,26}$ Only 2 studies reported low risk of bias for sequence generation and allocation concealment, although subjects in 10 out of 12 studies appeared to have similar baseline characteristics. Summary risk of bias within the studies, based on the risk of bias of key domains, showed that 5 studies were at low risk of bias; 2 were unclear; and 5 had high risk of bias in 1 or more key domains.

\section{Effects on Asthma Control}

Tools used to measure asthma control/severity among the included studies were the North of England asthma symptoms scale (score range 0-50), ${ }^{21}$ the Asthma Control Questionnaire (ACQ; score range 0-6), ${ }^{22,30}$ asthma morbidity index (score range 1-3), ${ }^{23}$ and asthma severity score (score range 5-15). ${ }^{28-29}$ Six studies reported the effects on asthma control in 957 patients (Figure 2). Of these studies, 3 were classified as having low risk of bias; 2 were high risk; and 1 had unclear risk of bias. The overall results indicated improvement of asthma control among patients who received self-management support service delivered by community pharmacists compared with those who received usual care (standardized mean difference $[\mathrm{SMD}]=0.46 ; 95 \% \mathrm{CI}=0.09-0.82)$, although substantial heterogeneity was observed $\left(I^{2}=82.6 \% ; P=0.000\right)$. Potential sources 


\section{TABLE 2 Characteristics of Asthma Self-Management Support and Other Services Among Included Studies}

\begin{tabular}{|c|c|c|c|c|c|c|c|c|c|}
\hline \multirow[b]{2}{*}{ Study } & \multirow[b]{2}{*}{$\begin{array}{l}\text { Pharmacist } \\
\text { Training }\end{array}$} & \multicolumn{2}{|c|}{$\begin{array}{c}\text { Self- } \\
\text { Management } \\
\text { Support }\end{array}$} & \multicolumn{3}{|c|}{$\begin{array}{l}\text { Education and } \\
\text { Skill Training }\end{array}$} & \multirow[b]{2}{*}{ Others } & \multirow[b]{2}{*}{$\begin{array}{l}\text { Service Length and } \\
\text { Schedule of Intervention/ } \\
\text { Follow-up Visit }\end{array}$} & \multirow[b]{2}{*}{$\begin{array}{c}\text { Compliance of } \\
\text { Pharmacist to Protocol }\end{array}$} \\
\hline & & 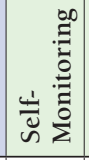 & 莺 $\frac{\pi}{\pi}$ & 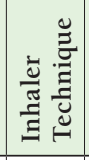 & 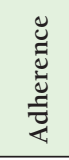 & 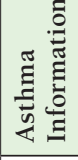 & & & \\
\hline $\begin{array}{l}\text { Armour } \\
(2007)^{20}\end{array}$ & $\begin{array}{l}\text { Yes, 2-day course on asthma, risk } \\
\text { assessment, the NAC manage- } \\
\text { ment plan, }{ }^{b} \text { patient education, } \\
\text { goal setting, adherence assess- } \\
\text { ment, and spirometry }\end{array}$ & NR & Yes & Yes & Yes & Yes & $\begin{array}{l}\text { Identify DTP and } \\
\text { referral to GP }\end{array}$ & $\begin{array}{l}\text { NR; baseline then follow- } \\
\text { up visit at } 1,3 \text { (optional), } \\
\text { and } 6 \text { months }\end{array}$ & $\begin{array}{l}\text { NR; visits and contact } \\
\text { regularly via phone by } \\
\text { project staff and news- } \\
\text { letter }\end{array}$ \\
\hline $\begin{array}{l}\text { Barbanel } \\
(2003)^{21}\end{array}$ & Yes, 3-day course on asthma care & Yes & Yes & Yes & NR & Yes & $\begin{array}{l}\text { Smoking } \\
\text { cessation }\end{array}$ & $\begin{array}{l}45-60 \text { minutes, then } \\
\text { follow-up every week by } \\
\text { phone for } 3 \text { months }\end{array}$ & NR \\
\hline $\begin{array}{l}\text { Charrois } \\
(2006)^{22}\end{array}$ & $\begin{array}{l}\text { Yes, focused on self-management, } \\
\text { appropriate drug therapy, and } \\
\text { monitoring }\end{array}$ & NR & Yes & Yes & NR & Yes & $\begin{array}{l}\text { Optimization of } \\
\text { drug therapyc }\end{array}$ & $\begin{array}{l}\text { NR; baseline then follow- } \\
\text { up visit at } 2 \text { weeks and at } \\
1,2,4 \text {, and } 6 \text { months }\end{array}$ & $\begin{array}{c}\text { Low, in some aspects of } \\
\text { intervention }\end{array}$ \\
\hline $\begin{array}{l}\text { Herborg } \\
(2001)^{23}\end{array}$ & $\begin{array}{l}\text { Yes, a self-study manual and } \\
\text { 2-day course on asthma manage- } \\
\text { ment and TOM with simulated } \\
\text { patient care }\end{array}$ & Yes & Yes & Yes & Yes & Yes & $\begin{array}{l}\text { Identify and } \\
\text { analyze DTP }\end{array}$ & $\begin{array}{l}41 \text { minutes on average; } \\
\text { visit once a month for } \\
12 \text { months }\end{array}$ & $\begin{array}{c}\text { NR; participating } \\
\text { pharmacists met every } \\
3 \text { months }\end{array}$ \\
\hline $\begin{array}{l}\text { Kheir } \\
(2001)^{24}\end{array}$ & $\begin{array}{l}\text { Yes, trained in asthma manage- } \\
\text { ment and provision of the service }\end{array}$ & Yes & Yes & Yes & Yes & Yes & $\begin{array}{l}\text { Identify and } \\
\text { resolve DTP }\end{array}$ & $\begin{array}{l}\text { NR; visit once a month or } \\
\text { as needed for } 4 \text { months }\end{array}$ & NR \\
\hline $\begin{array}{l}\text { McLean } \\
(2003)^{25}\end{array}$ & $\begin{array}{l}\text { Yes, trained in pharmaceuti- } \\
\text { cal care on asthma (with } 80 \% \\
\text { requirement on examination and } \\
3 \text { submitted cases) }\end{array}$ & Yes & Yes & Yes & Yes & Yes & NR & $\begin{array}{l}1 \text { hour every } 2-3 \text { weeks for } \\
\text { at least } 3 \text { appointments, } \\
\text { then follow-up every } \\
3 \text { months for the remainder } \\
\text { of the study ( } 12 \text { months) }\end{array}$ & NR \\
\hline $\begin{array}{l}\text { Petkova } \\
(2008)^{26} \\
\end{array}$ & Yes & Yes & Yes & Yes & Yes & Yes & $\begin{array}{l}\text { Smoking } \\
\text { cessation }\end{array}$ & $\begin{array}{l}\text { NR; visit once a month for } \\
4 \text { months }\end{array}$ & NR \\
\hline $\begin{array}{l}\text { Saini } \\
(2004)^{27}\end{array}$ & $\begin{array}{l}\text { Yes, } 2 \text {-day course based on the } \\
\text { NAC management plan }\end{array}$ & Yes & Yes & Yes & Yes & Yes & NR & $\begin{array}{l}56.6 \text { minutes at baseline } \\
\text { then follow-up visit at } 1 \\
\text { month (18.8 min), } \\
3 \text { months ( } 21.1 \mathrm{~min}) \text { and } \\
6 \text { months (collect endpoint) } \\
\end{array}$ & $\begin{array}{c}\text { NR; a service audit was } \\
\text { conducted through } \\
\text { observation }\end{array}$ \\
\hline $\begin{array}{l}\text { Saini } \\
(2008)^{28}\end{array}$ & $\begin{array}{l}\text { Yes, based on the NAC manage- } \\
\text { ment plan with a focus on local } \\
\text { asthma issues }\end{array}$ & Yes & Yes & Yes & Yes & Yes & NR & $\begin{array}{l}41.2+11.5 \text { minutes at base- } \\
\text { line then follow-up visit at } \\
1,3 \text { (optional), and } \\
6 \text { months ( } 15.6+7.2 \text { min- } \\
\text { utes at the final visit) }\end{array}$ & NR \\
\hline $\begin{array}{l}\text { Schulz } \\
(2001)^{29}\end{array}$ & $\begin{array}{l}\text { Yes, asthma-related knowledge } \\
\text { (5 hours), communication skills } \\
\text { (6 hours), and study protocol } \\
\text { ( } 2 \text { hours) }\end{array}$ & Yes & NR & Yes & Yes & NR & $\begin{array}{l}\text { Detected and } \\
\text { solved DTP or } \\
\text { health-related } \\
\text { problems }\end{array}$ & $\begin{array}{l}\text { Every } 6 \text { weeks for } \\
12 \text { months (overall } 9 \text { visits) }\end{array}$ & NR \\
\hline $\begin{array}{l}\text { Smith } \\
(2007)^{30}\end{array}$ & $\begin{array}{l}\text { Yes, l-day course to facilitate } \\
\text { patients' self-management behav- } \\
\text { ior, use of material aids and com- } \\
\text { munication skills }\end{array}$ & $\mathrm{U}^{\mathrm{d}}$ & Yes & $\mathrm{U}^{\mathrm{d}}$ & $\mathrm{U}^{\mathrm{d}}$ & $\mathrm{U}^{\mathrm{d}}$ & NR & $\begin{array}{l}\text { Approximately } 45 \text { minutes } \\
\text { at baseline then follow-up } \\
\text { visit at } 1,2,4,6 \text {, and } 9 \\
\text { months ( } 20 \text { minutes for } \\
\text { follow-up visits) }\end{array}$ & $\begin{array}{c}\text { NR; regular contact } \\
\text { by researchers via site } \\
\text { visits, phone calls, and } \\
\text { newsletters }\end{array}$ \\
\hline $\begin{array}{l}\text { Stergachis } \\
(2002)^{31}\end{array}$ & $\begin{array}{l}\text { Yes, 8-hour course to cover } \\
\text { asthma management and tips for } \\
\text { communicating with children }\end{array}$ & Yes & Yes & Yes & NR & Yes & $\begin{array}{l}\text { Identify and } \\
\text { resolve DTP }\end{array}$ & \begin{tabular}{|l|}
$26.8+18.2$ minutes, \\
every refill process for \\
12 months \\
\end{tabular} & Low \\
\hline \multicolumn{10}{|c|}{ 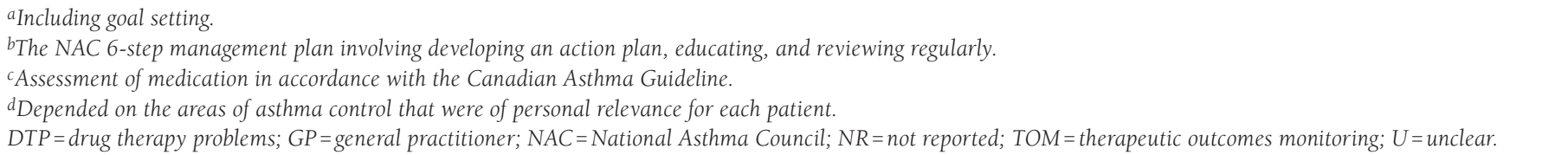 } \\
\hline
\end{tabular}




\section{FIGURE 2 Forest Plot of the Effect of Asthma Self-Management Support Service Provided by} Community Pharmacist

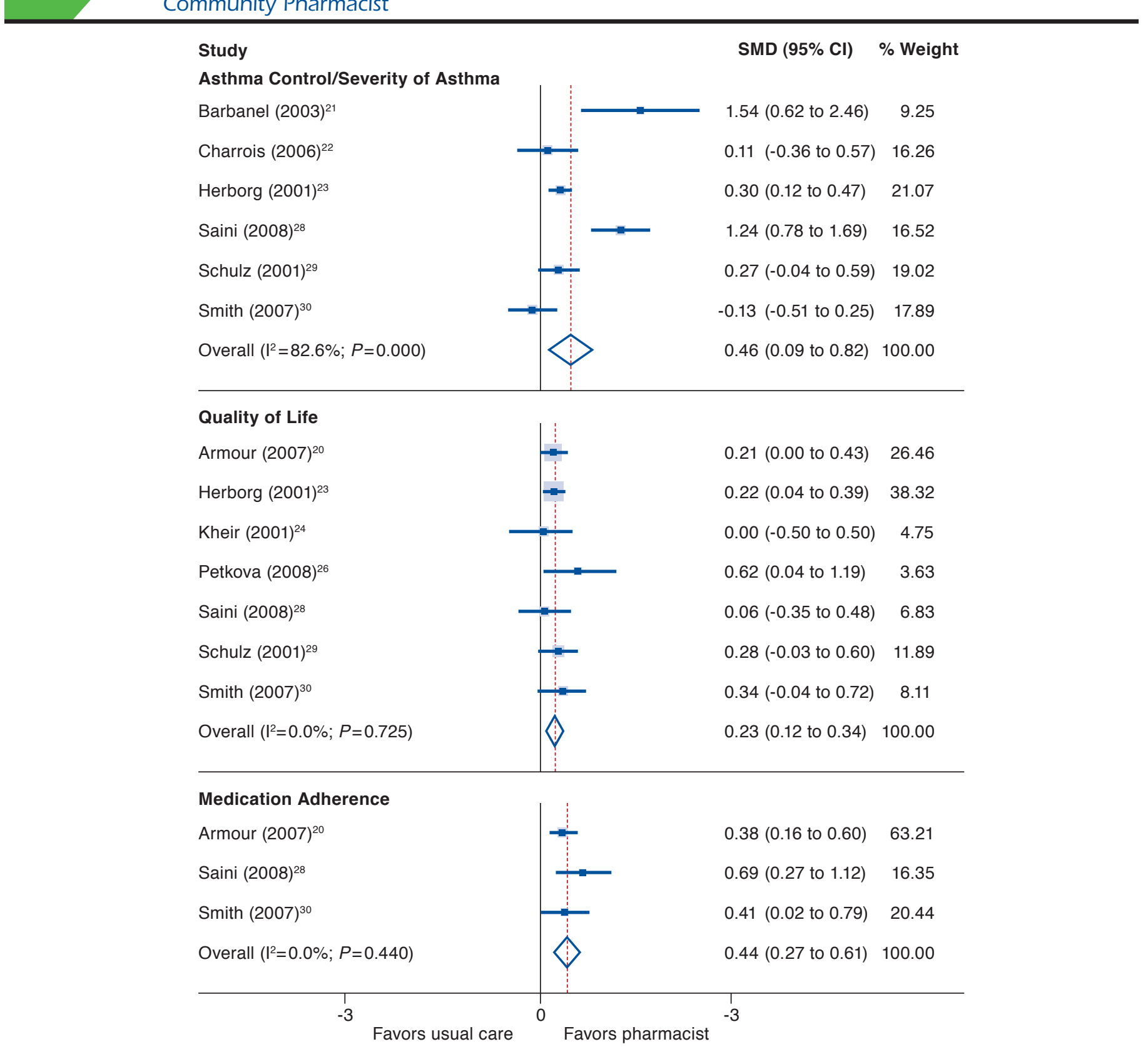

Note: Weights are from random effects analysis.

$C I=$ confidence interval; $S M D=$ standardized mean difference.

of heterogeneity were investigated and showed no clear source of heterogeneity.

Findings from subgroup meta-analyses are presented in Table 3. These results indicate significant benefit of a self-management support service on asthma control in the following subgroups: patients with uncontrolled asthma (SMD $=0.71$; 95\% CI=0.13-1.29); service intervention that was composed of both self-monitoring support and action plan provision ( $\mathrm{SMD}=0.96 ; 95 \% \mathrm{CI}=0.14-1.78)$; short study duration of 6 months or less (SMD $=0.92 ; 95 \% \mathrm{CI}=0.03-1.81$ ); and having 
TABLE 3 Effect of Asthma Self-Management Support Service Provided by Community Pharmacist in Subgroup Meta-Analysis

\begin{tabular}{|c|c|c|c|c|c|c|}
\hline \multirow[b]{2}{*}{ Subgroup Variables } & \multicolumn{3}{|c|}{ Asthma Control/Severity } & \multicolumn{3}{|c|}{ Quality of Life } \\
\hline & Summary SMD & $\begin{array}{c}\text { Heterogeneity, } \\
\%\end{array}$ & $\begin{array}{c}\text { Studies } \\
\text { (Patients), } \mathrm{n}\end{array}$ & Summary SMD & $\begin{array}{c}\text { Heterogeneity, } \\
\%\end{array}$ & $\begin{array}{c}\text { Studies } \\
\text { (Patients), } \mathrm{n}\end{array}$ \\
\hline \multicolumn{7}{|l|}{ Patients' asthma condition } \\
\hline Uncontrolled asthma & 0.71 (0.13 to 1.29$)$ & $86.2(P=0.000)$ & $4(684)^{21-23,28}$ & $0.19(0.06$ to 0.31$)$ & $0.0(P=0.789)$ & $4(1,048)^{20,23,24,28}$ \\
\hline Any level of asthma & $0.09(-0.31$ to 0.48$)$ & $61.4(P=0.108)$ & $2(273)^{29,30}$ & $0.35(0.13$ to 0.58$)$ & $0.0(P=0.603)$ & $3(323)^{26,29,30}$ \\
\hline \multicolumn{7}{|l|}{ Service component } \\
\hline Self-monitoring and action plan & $0.96(0.14$ to 1.78$)$ & $89.9(P=0.000)$ & $3(614)^{21,23,28}$ & $0.20(0.12$ to 0.34$)$ & $3.2(P=0.376)$ & $4(702)^{23,24,26,28}$ \\
\hline Self-monitoring or action plan & $0.10(-0.15$ to 0.35$)$ & $22.8(P=0.274)$ & $3(343)^{22,29,30}$ & $0.25(0.09$ to 0.41$)$ & $0.0(P=0.836)$ & $3(669)^{20,29,30}$ \\
\hline \multicolumn{7}{|l|}{ Study duration } \\
\hline$\leq 6$ months & $0.92(0.03$ to 1.81$)$ & $86.2(P=0.001)$ & $3(184)^{21,22,28}$ & $0.20(0.02$ to 0.37$)$ & $2.8(P=0.379)$ & $4(598)^{20,24,26,28}$ \\
\hline$>6$ months & $0.19(-0.04$ to 0.42$)$ & $51.2(P=0.129)$ & $3(773)^{23,29,30}$ & $0.25(0.10$ to 0.39$)$ & $0.0(P=0.827)$ & $3(773)^{23,29,30}$ \\
\hline \multicolumn{7}{|l|}{ Additional service } \\
\hline Identify and resolve DTP & $0.29(0.14$ to 0.45$)$ & $0.0(P=0.902)$ & $2(664)^{23,29}$ & 0.21 (0.09 to 0.33$)$ & $0.0(P=0.829)$ & $4(1,122)^{20,23,24,29}$ \\
\hline No DTP-related service & $0.63(-0.14$ to 1.41$)$ & $89.3(P=0.000)$ & $4(293)^{21,22,28,30}$ & $0.30(0.01$ to 0.58$)$ & $18.5(P=0.293)$ & $3(249)^{26,28,30}$ \\
\hline
\end{tabular}

an additional service involving identifying and resolving DTPs $(\mathrm{SMD}=0.29 ; 95 \% \mathrm{CI}=0.14-0.45)$.

\section{Effects on Quality of Life}

Six measures were used to evaluate quality of life, which included the Asthma Quality of Life Questionnaire (AQLQ), ${ }^{20,24,30}$ the Nottingham Health Profile (NHP), ${ }^{23}$ the Living with Asthma Questionnaire (LWAQ), ${ }^{23,29}$ the Short Form-36, ${ }^{24,29}$ asthma assessment form, ${ }^{26}$ and asthma-related QOL score. ${ }^{28}$ Scales of the used measures varied and ranged from 1-3 for LWAQ to 20-100 for asthma-related QOL score. Pooled estimates of selfmanagement support service among 7 studies involving 1,371 patients showed significant improvement in quality of life of asthma patients (SMD $=0.23 ; 95 \% \mathrm{CI}=0.12-0.34)$ with no heterogeneity $\left(\mathrm{I}^{2}=0.00 \% ; P=0.725\right) .20,23,24,26,28-30$ Increase in quality of life among self-management support groups was present in all subgroup populations, although relatively higher SMDs were observed in the following subgroups (Table 3): patients with any level of asthma ( $\mathrm{SMD}=0.35 ; 95 \% \mathrm{CI}=0.13-0.58)$; service intervention that comprised either self-monitoring support or action plan provision ( $\mathrm{SMD}=0.25 ; 95 \% \mathrm{CI}=0.09$ 0.41 ); study duration of more than 6 months (SMD $=0.25 ; 95 \%$ $\mathrm{CI}=0.10-0.39)$; and having no additional service related to DTP $(\mathrm{SMD}=0.30 ; 95 \% \mathrm{CI}=0.01-0.58)$.

\section{Effects on Adherence}

Adherence measures used in the studies included the Brief Medication Questionnaire (BMQ) and Medication Adherence Report Scale (MARS), 20,28,30 which are self-administered questionnaires that measure participants' behavior regarding the use of medicines. Findings in all 3 studies (595 patients) showed significant improvement in medication adherence among patients with asthma who received self-management support. Correspondingly, the pooled estimates of SMD in the self-management support group were higher than those in the usual care group ( $\mathrm{SMD}=0.44 ; 95 \%$ $\mathrm{CI}=0.27-0.61)$ without heterogeneity $\left(I^{2}=0.0 \% ; P=0.440\right)$. No subgroup analysis was conducted in studies that reported effects on adherence as there were fewer than 2 studies in all subgroup categories.

\section{Discussion}

Although the concept of supported asthma self-management is universal, its effects when provided in different settings may vary due to the competency of the service provider, accessibility and facility of the setting, as well as patients' trust in providers. To the best of our knowledge, this is the first meta-analysis that has studied the effects of pharmacy services provided by community pharmacists to support asthma self-management. Findings from this analysis have strengthened the evidence for the beneficial effects of asthma self-management support services. Community pharmacy services that engaged the concept and components of supported self-management have been shown to improve asthma control, quality of life, and medication adherence in adult patients with asthma. Specifically, the positive effect of this service on asthma control was higher in studies of short duration (i.e., 6 months or less) in patients who had poorly controlled asthma, received training/support in self-monitoring and following an action plan, and received an additional service to identify and resolve DTPs.

A recent systematic meta-review found 2 previous systematic reviews that studied components of supported asthma self-management. ${ }^{7}$ According to these reviews, it was suggested that optimal self-management education should include advice on self-monitoring and a written action plan that was 
supported by regular professional review. ${ }^{6}$ Reducing the intensity of self-management education or level of clinical review may also reduce its effectiveness. ${ }^{32}$ Findings in the present review have reinforced that self-management support offered through guided self-monitoring and an asthma action plan provision were crucial to successfully achieve effectiveness in asthma control.

Findings from a recent systematic review of community pharmacy services showed that interventions that contributed to the improvement of asthma control and quality of life included face-to-face or computer training on inhalation technique, printed or mailed information to personalized programs, phone calls, and regular follow-up. ${ }^{11}$ In addition to these interventions, the pooled estimates identified in the present study showed that community pharmacy self-management support service significantly improved asthma control. However, results from a subgroup analysis indicated that this service could provide a significant improvement in asthma control only when combined with other pharmaceutical care elements, namely, resolution of DTPs. The effect of this added element was well aligned with findings from a current randomized controlled trial that the combined service of identifying pharmaceutical care issues and medicines use review help improve asthma control in adult patients. ${ }^{33}$

Considering other secondary outcomes, it was not surprising to observe similar beneficial effects of community pharmacy asthma self-management support on patients' quality of life and their medication adherence. This is because quality of life and medication adherence have been shown to correlate well with asthma control. ${ }^{34,35}$ Although it is interesting to explore if new service models would negatively affect patients' behavior and patterns of health care utilization, none of the included studies reported effects on these aspects. We suggest that future clinical studies on effects of service intervention should not focus only on the effectiveness; any potential unwanted outcomes in other perspectives should also be considered and reported.

Other common missing information from service intervention trials was the fidelity of service providers to the study protocol, which may have had a substantial effect on the measured outcomes. In this review, few studies reported or mentioned fidelity to study protocol, and a small number of studies described how to support or encourage participating pharmacists to comply with the study protocol. Among the 6 studies that were used to estimate pooled effects on asthma control, 1 reported that participating pharmacists did not comply with the study protocol. ${ }^{22}$ Interestingly, the findings from this study showed no difference in asthma control between patients who received the self-management support service and those who received usual care. ${ }^{22}$ If effective strategies had been implemented to ensure fidelity to protocol of participating pharmacists, it may have changed not only the findings of that particular study but also the overall estimates on asthma control in this review.

Asthma self-management requires a certain degree of health literacy and self-care competency. However, none of the included studies for meta-analysis had specifically targeted disadvantaged populations such as those with psychiatric problems, decrements in mental ability, learning disabilities, physical disabilities (e.g., hearing, speech, or vision impairments), culturally and linguistically diverse populations, indigenous populations, or young children. Hence, the effectiveness of selfmanagement support services found in this study may not be applicable to those patient groups. It should also be noted that all of the included studies were conducted in Western countries. The pooled effects showed in this study may be altered if the service were implemented in developing countries where there are differences in practice guidelines, organization of health care/health care structure, and attitudes toward asthma self-management among patients and health care practitioners, as well as varying interest and capacity among community pharmacists to deliver services, with respect to time and staffing availability, and lack of reimbursement of such services. Implementation of any recommended effective services into practice is a challenge for the profession worldwide and is worth exploring further to gain more knowledge and understanding on barriers and facilitators to support the implementation of such services.

\section{Limitations}

Despite rigorous study design and comprehensive searching, this review experienced some limitations, which included the use of SMD, heterogeneity, and quality among the included studies. There is no unit for SMD, which makes it hard to interpret the pooled estimates in this study. However, reporting SMD was the only valid approach because of the variation in tools that measured outcomes of interests on a different scale and were used in the included studies.

A specific service intervention (i.e., support for asthma selfmanagement) was selected from other community pharmacy services in an attempt to reduce clinical heterogeneity among included studies. Yet statistical heterogeneity of studies on asthma control was still observed.

Factors that could explain inconsistent responses on asthma control between studies include a variation in tools used to measure asthma control/severity, as well as treatment practice and medicines prescribed. It should be noted that nearly half of the included studies were at high risk of bias. However, they are the only relevant evidence available in this area. Caution should therefore be exercised when interpreting findings in this meta-analysis, since bias among the included studies could affect their reported outcomes, which would consequently influence the pooled estimates identified. Consequently, future research should be conducted with rigorous methods to avoid 
potential risk of bias, particularly contamination of service intervention, by using a cluster randomization approach. A test for publication bias was planned but could not be done because of the small number of studies for each outcome.

\section{Conclusions}

This meta-analysis gathered relevant evidence and quantified the pooled estimates to consolidate evidence for the benefits of services containing self-management support provided by community pharmacists in helping patients with asthma to improve their asthma control as well as quality of life and adherence to medication. In an attempt to expand their role in asthma care, community pharmacists should include selfmanagement support components into their general pharmaceutical care service. However, further well-designed clinical studies are needed to strengthen findings from the current evidence.

\section{Authors}

SUTHASINEE DOKBUA, PharmD, and TEERAPON DHIPPAYOM, PharmD, PhD, Faculty of Pharmaceutical Sciences, Naresuan University, Phitsanulok, Thailand. PIYAMETH DILOKTHORNSAKUL, PharmD, PhD, Center of Pharmaceutical Outcomes Research, Department of Pharmacy Practice, Faculty of Pharmaceutical Sciences, Naresuan University, Phitsanulok, Thailand. NATHORN CHAIYAKUNAPRUK, PharmD, PhD, Center of Pharmaceutical Outcomes Research, Department of Pharmacy Practice, Faculty of Pharmaceutical Sciences, Naresuan University, Phitsanulok, Thailand; School of Pharmacy, Monash University Malaysia, Selangor, Malaysia; School of Pharmacy, University of Wisconsin, Madison; and Asian Centre for Evidence Synthesis in Population, Implementation and Clinical Outcomes, Health and Well-being Cluster, Global Asia in the 21st Century Platform, Monash University Malaysia, Selangor. BANDANA SAINI, PhD, and INES KRASS, BPharm, Dip Hosp Pharm, Grad Dip Educ Studies (Health Ed), PhD, Faculty of Pharmacy, the University of Sydney, Australia.

AUTHOR CORRESPONDENCE: Teerapon Dhippayom, PharmD, PhD, Faculty of Pharmaceutical Sciences, Naresuan University, Phitsanulok, Thailand 65000. Tel.: 66.55.961.839;

E-mail: teerapond@nu.ac.th.

\section{REFERENCES}

1. World Health Organization. Asthma. 2017. Available at: http://www.who. int/respiratory/asthma/en/. Accessed September 13, 2018.

2. Global Initiative for Asthma. Global strategy for asthma management and prevention. 2018. Available at: https://ginasthma.org/2018-gina-report-global-strategy-for-asthma-management-and-prevention/. Accessed September 13, 2018.

3. National Asthma Council Australia. Asthma handbook - quick reference guide, version 1.2. 2016. Available at: http://www.asthmahandbook.org.au. Accessed September 13, 2018.

4. National Institute for Health and Care Excellence. Asthma: NICE quality standard 25. 2013. Available at: https://www.nice.org.uk/guidance/qs25. Accessed September 13, 2018.

5. Denford S, Taylor RS, Campbell JL, Greaves CJ. Effective behavior change techniques in asthma self-care interventions: systematic review and metaregression. Health Psychol. 2014;33(7):577-87.

6. Gibson PG, Powell H, Coughlan J, et al. Self-management education and regular practitioner review for adults with asthma. Cochrane Database Syst Rev. 2003;(1):Cd001117.

7. Pinnock H, Parke HL, Panagioti M, et al. Systematic meta-review of supported self-management for asthma: a healthcare perspective. BMC Med. 2017;15(1):64

8. García-Cárdenas V, Sabater-Hernández D, Kenny P, Martínez-Martínez F, Faus MJ, Benrimoj SI. Effect of a pharmacist intervention on asthma control. A cluster randomised trial. Respir Med. 2013;107(9):1346-55.

9. Armour CL, Reddel HK, LeMay KS, et al. Feasibility and effectiveness of an evidence-based asthma service in Australian community pharmacies: a pragmatic cluster randomized trial. J Asthma. 2013;50(3):302-09.

10. Fathima M, Naik-Panvelkar P, Saini B, Armour CL. The role of community pharmacists in screening and subsequent management of chronic respiratory diseases: a systematic review. Pharm Pract (Granada). 2013;11(4):228-45.

11. Senna G, Caminati M, Bovo C, Canonica GW, Passalacqua G. The role of the pharmacy in the management of bronchial asthma: A literature-based evaluation. Ann Allergy Asthma Immunol. 2017;118(2):161-65.

12. Cochrane Effective Practice and Organisation of Care. 2017. Available at: http://epoc.cochrane.org/epoc-specific-resources-review-authors. Accessed September 13, 2018.

13. Moher D, Liberati A, Tetzlaff J, Altman DG. Preferred reporting items for systematic reviews and meta-analyses: the PRISMA statement. Ann Intern Med. 2009;151(4):264-69, W64.

14. Cochrane Effective Practice and Organisation of Care. Good practice data collection form. EPOC resources for review authors. 2013. Available at: http://epoc.cochrane.org/epoc-specific-resources-review-authors. Accessed September 13, 2018.

15. Cochrane Effective Practice and Organisation of Care. Suggested risk of bias criteria for EPOC reviews. EPOC resources for review authors. 2016. Available at: http://epoc.cochrane.org/epoc-specific-resources-reviewauthors. Accessed September 13, 2018.

16. Cochrane Effective Practice and Organisation of Care. Summary assessments of the risk of bias. EPOC resources for review authors. 2013. Available at: http://epoc.cochrane.org/epoc-specific-resources-review-authors. Accessed September 13, 2018.

17. Cochrane Effective Practice and Organisation of Care. Taking account of adverse effects in EPOC reviews. EPOC resources for review authors. 2013. Available at: http://epoc.cochrane.org/epoc-specific-resources-reviewauthors. Accessed September 13, 2018.

18. DerSimonian R, Laird N. Meta-analysis in clinical trials. Control Clin Trials. 1986;7(3):177-88.

19. Higgins JPT, Green S, eds. Cochrane handbook for systematic reviews of interventions, version 5.1.0: the Cochrane Collaboration. March 2011. Available at: http://handbook-5-1.cochrane.org/. Accessed September 13, 2018.
This study received financial support from Naresuan University's Faculty of Pharmaceutical Sciences Research Fund. Two authors, Saini and Krass, have studies that were included in this review. However, they were not involved in the processes that could bias outcomes of the present study, that is, quality assessment and meta-analysis. The remaining authors have declared no conflicts of interest. 
20. Armour C, Bosnic-Anticevich S, Brillant M, et al. Pharmacy Asthma Care Program (PACP) improves outcomes for patients in the community. Thorax. 2007;62(6):496-502.

21. Barbanel D, Eldridge S, Griffiths C. Can a self-management programme delivered by a community pharmacist improve asthma control? A randomised trial. Thorax. 2003;58(10):851-54.

22. Charrois TL, Newman SC, Senthilselvan A, Tsuyuki RT. Improving asthma control in the rural setting: the BREATHE (Better Respiratory Education and Asthma Treatment in Hinton and Edson) study. Can Pharm J (Ott). 2006;139(4):44-50

23. Herborg H, Soendergaard B, Froekjaer B, et al. Improving drug therapy for patients with asthma_part 1: patient outcomes. J Am Pharm Assoc. 2001;41(4):539-50.

24. Kheir N, Emmerton L, Shaw J. Can pharmacists influence the health-related quality of life of patients with asthma?: The New Zealand Pharmaceutical Care experience. J Sci Res Med Sci. 2001;3(2):69-75.

25. McLean W, Gillis J, Waller R. The BC Community Pharmacy Asthma Study: a study of clinical, economic and holistic outcomes influenced by an asthma care protocol provided by specially trained community pharmacists in British Columbia. Can Respir J. 2003;10(4):195-202.

26. Petkova VB. Pharmaceutical care for asthma patients: a community pharmacy-based pilot project. Allergy Asthma Proc. 2008;29(1):55-61.

27. Saini B, Krass I, Armour C. Development, implementation, and evaluation of a community pharmacy-based asthma care model. Ann Pharmacother. 2004;38(11):1954-60.
28. Saini B, Filipovska J, Bosnic-Anticevich S, Taylor S, Krass I, Armour C. An evaluation of a community pharmacy-based rural asthma management service. Aust J Rural Health. 2008;16(2):100-08.

29. Schulz M, Verheyen F, Mühlig S, et al. Pharmaceutical care services for asthma patients: a controlled intervention study. J Clin Pharmacol. 2001;4l(6):668-76.

30. Smith L, Bosnic-Anticevich SZ, Mitchell B, Saini B, Krass I, Armour C. Treating asthma with a self-management model of illness behaviour in an Australian community pharmacy setting. Soc Sci Med. 2007;64(7):1501-11.

31. Stergachis A, Gardner JS, Anderson MT, Sullivan SD. Improving pediatric asthma outcomes in the community setting: does pharmaceutical care make a difference? J Am Pharm Assoc (Wash). 2002;42(5):743-52.

32. Powell H, Gibson PG. Options for self-management education for adults with asthma. Cochrane Database Syst Rev. 2003;(1):Cd004107.

33. Manfrin A, Tinelli M, Thomas T, Krska J. A cluster randomised control trial to evaluate the effectiveness and cost-effectiveness of the Italian medicines use review (I-MUR) for asthma patients. BMC Health Serv Res. 2017;17(1):300.

34. Makhinova T, Barner JC, Richards KM, Rascati KL. Asthma controller medication adherence, risk of exacerbation, and use of rescue agents among Texas Medicaid patients with persistent asthma. J Manag Care Spec Pharm. 2015;21(12):1124-32. Available at: https://www.jmcp.org/doi/10.18553/ jmcp.2015.21.12.1124.

35. Li Z, Thompson LA, Gross HE, et al. Longitudinal associations among asthma control, sleep problems, and health-related quality of life in children with asthma: a report from the PROMIS Pediatric Asthma Study. Sleep Med. 2016;20:41-50. 


\section{APPENDIX Methodological Quality Assessment of Included Studies}

\begin{tabular}{|c|c|c|c|c|c|c|c|c|c|c|}
\hline \multirow[b]{2}{*}{ Study } & \multicolumn{10}{|c|}{ Domain } \\
\hline & 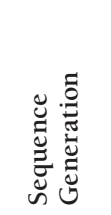 & 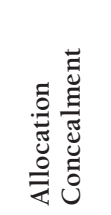 & 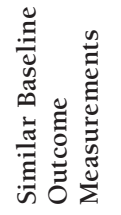 & 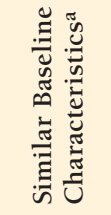 & 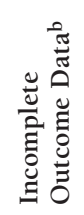 & 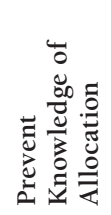 & 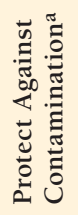 & 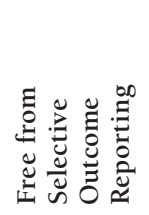 & 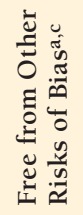 & 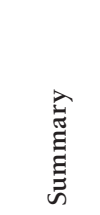 \\
\hline Armour (2007)20 & unclear & low & low & low & high & unclear & low & low & low & low \\
\hline Barbanel (2003)21 & unclear & high & low & low & high & unclear & high & low & low & high \\
\hline Charrois (2006) 22 & low & low & low & high & high & unclear & high & low & low & high \\
\hline Herborg $(2001)^{23}$ & high & high & high & unclear & high & unclear & low & low & low & unclear \\
\hline Kheir $(2001)^{24}$ & high & high & low & low & low & unclear & high & low & low & high \\
\hline McLean (2003) ${ }^{25}$ & low & low & low & unclear & high & high & low & unclear & low & unclear \\
\hline Petkova $(2008)^{26}$ & unclear & unclear & low & high & low & unclear & high & low & low & high \\
\hline Saini $(2004)^{27}$ & high & high & high & high & high & unclear & low & low & low & high \\
\hline Saini $(2008)^{28}$ & high & high & low & low & high & unclear & low & low & low & low \\
\hline Schulz (2001)29 & high & high & low & low & high & unclear & low & low & low & low \\
\hline Smith $(2007)^{30}$ & high & high & low & low & high & unclear & low & low & low & low \\
\hline Stergachis (2002) 31 & unclear & low & low & low & low & unclear & low & low & low & low \\
\hline
\end{tabular}

